\title{
A Distributed Face Recognition Framework Based on Data Fusion
}

\author{
Zheng Zhang ${ }^{1}$, Yan Guo ${ }^{2}$ and Guozhi Song ${ }^{1}$ \\ ${ }^{l}$ College of Computer Science and Software, Tianjin Polytechnic University, Tianjin, China, \\ 300387 \\ ${ }^{2}$ School of Computer Software, Tianjin University, Tianjin, China, 300072 \\ aaron_boy_2000@hotmail.com,812347622@qq.com,guozhi.song@googlemail.com
}

\begin{abstract}
To accomplish face recognition more efficiently, a distributed face recognition framework based on MB-LBP features and data fusion is presented in this paper. Firstly, four face regions are interactively marked and the Multi-scale Block Local Binary Patterns are extracted from these regions to achieve both locally and globally informative features. Secondly, a distributed framework is introduced to accelerate the recognition process, in which features of each single face region are utilized to perform face classification in parallel. The final decision is made by a kind of data fusion mechanism based on an artificial neural network (ANN) to make rational use of the confidence information got from the classification of each region. In experiment, the runtime and recognition performance of our system is compared with several other popular face recognition paradigms. The results indicate that the distributed framework presented in this paper can promote the efficiency of face recognition prominently while not losing accuracy in recognition performance.
\end{abstract}

Keywords: face recognition; distributed framework; MB-LBP; data fusion

\section{Introduction}

In light of advances in image and signal processing in recent years, various methods have been proposed for face recognition [1-5]. Holistic subspace method, such as PCA [6-8], ICA [9] and LDA [10] based techniques, have played a very important role in face recognition. Another approach is to construct a local appearance-based feature space, utilizing appropriate image filters, to make the distributions of faces less affected by various changes. Local features analysis (LFA) [11], and wavelet-based methods [12,13] are among these.

Local binary pattern (LBP) $[3,4]$ has been proved to be effective for image representation, but it is susceptible to noises and too local to be robust. In the definition of multi-block local binary patterns (MB-LBP) [5], the computation is based on average values of pixels in a neighborhood block instead of individual pixels. MB-LBP is more robust and can provide a more complete image representation than basic LBP in that it encodes not only microstructures but also macrostructures of image patterns.

However, the idea of multi-scale features usually yields high-dimensional feature vectors, which makes the subsequent classification process very time-consuming. So, in this paper we present a distributed framework based on MB-LBP features and data fusion. Firstly, four face regions are interactively marked and the multi-scale block local binary patterns are extracted from these regions to achieve both locally and globally informative features. Secondly, a distributed framework is introduced to accelerate the recognition process, in which features of each single face region are utilized to perform face classification in parallel. In $[14,15]$ the concept of Gabor fusion is introduced and the classification result given by PCA based 
method is synthesized by neural network to make the ultimate decision. Inspired by the data fusion system in [15], the final decision is made by a reasonable fusion of the confidence information got from the classification of each face region based on an artificial neural network. Experimental results show the improvement in efficiency of our system to some other popular face recognition paradigms.

The rest of this paper is organized as follows: In Section 2, we will have a short discussion about the CMU Pose, Illumination, and Expression database. Section 3 presents the MB-LBP composite features. And then in Section 4, a neural network is introduced to make a fusion of the confidence information got from the classification of each region by support vector machines. A distributed framework is presented in Section 5 to implement our face recognition system. Several experiments are conducted to verify the efficiency of our system in Section 6 and some significative conclusions are drawn in the last section.

\section{Face Database}

The CMU Pose, Illumination, and Expression (PIE) database [16] contains 41368 images of 68 subjects collected between October 2000 and December 2000. In our experiment, we randomly select 30 subjects. Our dataset contains the images of their frontal faces under 24 different illumination variations with the room lights switched on, as well as 3 frontal face images with different expressions.

In preprocessing, we interactively marked four face regions as shown in Figure 1. Since LBP is computed based on neighborhood pixels, there are overlaps between these face regions.

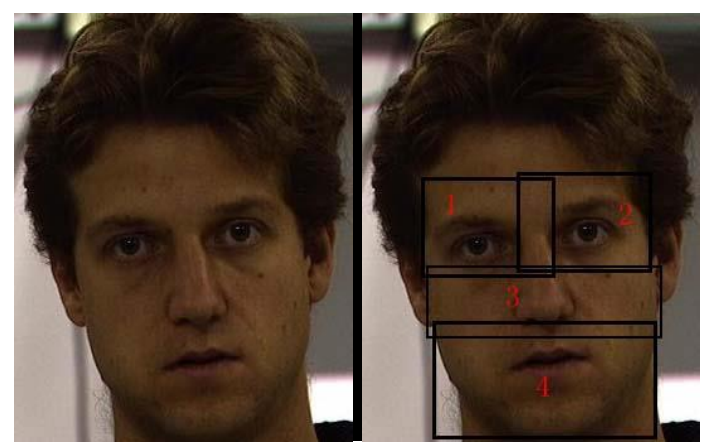

Figure 1. The Original Image From CUM PIE Database and Four Face Regions

Figure 2 shows some sample images from the dataset of our experiments with different illumination variations. 


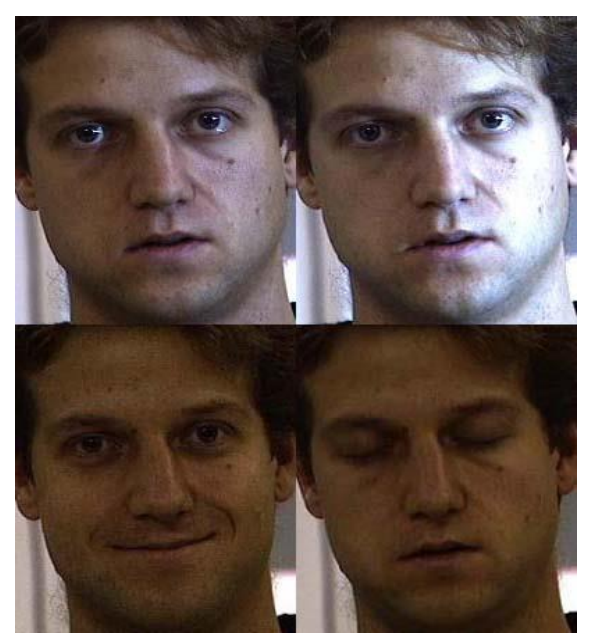

Figure 2. Sample Images with Different Illumination Variations

\section{Feature Extraction}

In this paper, MB-LBP features are extracted from each face regions to form both locally and globally informative composite features.

\subsection{MB-LBP}

Local binary patterns have been applied in many areas for the excellent capability of encoding local textures. The traditional LBP operator labels the pixels of an image by thresholding the $\mathrm{n} \times \mathrm{n}$ neighborhood of each pixel with the center value and considering the result as a binary number. Then the histogram of the labels can be used as a texture descriptor. Figure 3 shows the basic LBP operator.

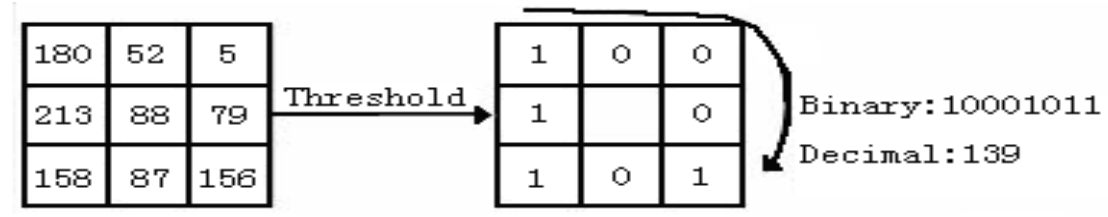

Figure 1. The Basic LBP Operator

Later the basic LBP descriptor was improved to use different shapes of neighborhoods [4]. Using circular neighborhoods and bilaterally interpolation allow any radius and number of pixels in the neighborhoods. Figure 4 gives an illustration of the circular neighborhood containing 8 pixels in it with radius equals 2 .

A big enhancement of LBP is the definition of uniform patterns [4]. A LBP descriptor is called uniform if the binary pattern contains at most two bitwise transitions from 0 to 1 or vice versa when the bit pattern is considered circular. In the computation of the LBP histogram, uniform patterns are used so that the histogram has a separate bin for every uniform pattern and all non-uniform patterns are assigned to a single bin, which simplify the standard LBP code greatly.

The following notation is used for the LBP operator: ${ }_{L B P}{ }_{P, R}{ }^{2}$. Where Superscript $\mathrm{u} 2$ stands for using only uniform patterns and the subscript represents using the operator in a $(\mathrm{P}, \mathrm{R})$ 
neighborhood. (P, R) is used for pixel neighborhood which means $\mathrm{P}$ sampling points on a circle of radius of $\mathrm{R}$.

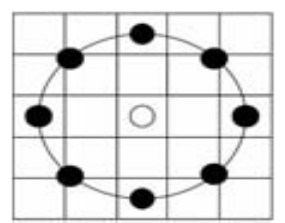

Figure 2. $\operatorname{LBP} \underset{8,2}{u 2}$, The Pixel Values are Bilaterally Interpolated Whenever the

\section{Sampling Point is Not in the Center of a Pixel}

However, the LBP operators discussed above are too local to be robust. In [5], multi-scale block LBP is introduced to encode both locally and globally informative features. In MBLBP, the comparison operator between single pixels in LBP is simply replaced with comparison between average pixel values of sub-blocks, as illustrated in Figure 5. We achieve better performance when utilizing multi-scale block for ${ }_{L B P}{ }_{8,2}{ }^{2}$ than for the basic $3 \times 3$ LBP operator. In this paper, the notation ${ }_{M B}{ }_{S}-L_{B P}{ }_{8,2}{ }^{\prime 2}$ is used for the Multi-scale Block ${ }_{L B P_{8,2}}{ }^{2}$ with the block size $S$.

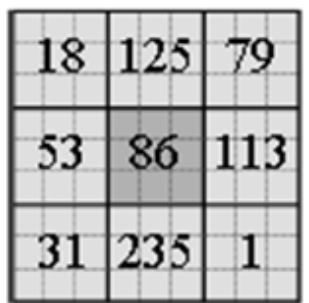

(a) Basic $3 \times 3$ MB-LBP Operator

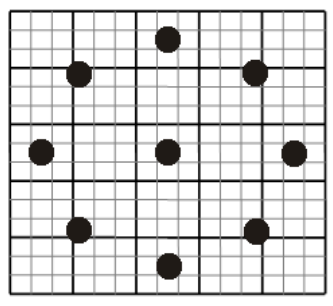

(b) MB- ${ }_{\text {LBP }}^{42} \underset{8,2}{\text { Op }}$ Operator

Figure 5. Basic $3 \times 3$ MB-LBP and MB- ${ }_{-18 P}^{42}$

Figure 6 gives some ${ }_{M B}{ }_{S}-L B P_{8,2}{ }^{2}$ filtered images of a face in our dataset.

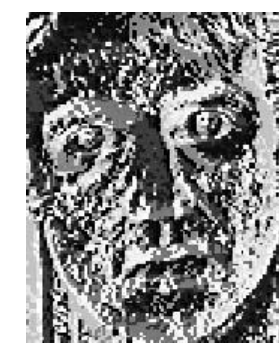

(a)

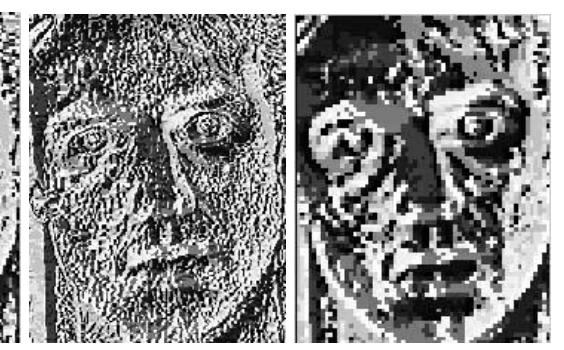

(b)

\begin{abstract}
(c)
\end{abstract}

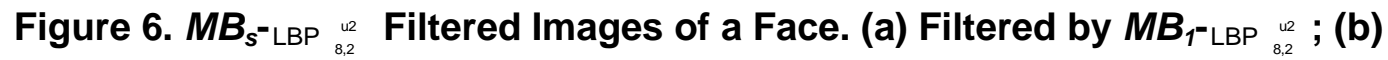

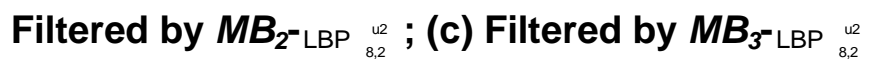




\subsection{Partitions}

We preserve both global and local features by partitioning an image into $P \times Q$ distinct windows. The number of partitions is determined by the scale at which the LBP features are extracted. Specifically, at the coarsest scale with relatively big LBP block, less partitions for each face region is sufficient because the coarse filter extracts only global structures, while at a finer scale, usually more partitions are needed for each face region. And since different LBP block sizes are used, in each partition the number of LBP blocks is roughly the same. Here, the ${ }_{M B}{ }_{S}-L_{B P}{ }_{8,2}^{\prime 2}$ operator with about $10 \times 15$ blocks is selected since it is a good trade-off between recognition performance and feature vector length.

\subsection{MB-LBP Composite Features}

The MB-LGBP features can be achieved within the following two steps:

(1) Utilize MB-LBP with block size $S=1,2,3$ to encode face region images.

(2) Concatenate global and local MB-LBP features extracted from all partitions of each face region at all scales to form the composite features $\Phi$.

\section{Classification}

In this paper, the classification process is actually done in 2 steps. Firstly, the confidence information of one sample belonging to each class is computed by SVM. And then, a reasonable fusion of the confidence information is made by an artificial neural network.

\subsection{SVM Classification}

In classification of each face region, C-SVM [17-19] with radial basis kernel (RBF) is used as classifier. However, SVM is suitable only for binary classification, while our face classification is a 30-class problem. There are many techniques that can extend SVM to handle multi-class problems, such as "one-against-one" voting strategy, "one-againstone" eliminating strategy, and "one-against-all" max response strategy. In this paper we utilize the "one-against-all" max response strategy, which can be summarized as follows: We train $N$ ( $N$ equals to 30, the number of subjects in our dataset) classifiers $h_{l}, h_{2}, \ldots$, and $h_{N}$. For classifier $h_{i}$, sample images belonging to class $_{i}$ are positive, while the rest are negative. First, the sample $\vec{x}$ is classified by all the $N$ classifiers, suppose the outputs are $f_{1}(\vec{x}), f_{2}(\vec{x}), \ldots, f_{N}(\vec{x})$. Then the final decision is set to $\operatorname{argmax}\left(f_{i}(\vec{x})\right), 1<=i<=N$, and the value of $f_{i}(\vec{x})$ can be regarded as the confidence of $\vec{x}$ belonging to class ${ }_{i}$.

By computing the confidence information of a sample belonging to $N$ classes using each face region, we get its confidence table. Table 1 gives part of the confidence information of image 04060/lights/27-00.

Table 1. Face Region Confidence Table for Image 04060/lights/27-00

\begin{tabular}{lllllll}
\hline Cl & 04002 & 04013 & 04037 & 04058 & 04060 & 04063 \\
$\begin{array}{l}\text { ass } \\
\text { Face } \\
\text { Region }\end{array}$ & & & & & & \\
\hline Region 1 & 0.0781 & 0.4672 & 0.5322 & 0.2134 & 0.6481 & 0.1526 \\
\hline Region 2 & 0.0862 & 0.5417 & 0.3915 & 0.3153 & 0.8458 & 0.0087 \\
\hline Region 3 & 0.2864 & 0.6332 & 0.2328 & 0.1360 & 0.2451 & 0.1622 \\
\hline Region 4 & 0.1625 & 0.2404 & 0.4649 & 0.0698 & 0.5412 & 0.0476 \\
\hline
\end{tabular}




\subsection{Data Fusion Based on Artificial Neural Network}

The final decision can be obtained by an artificial neural network [20] based data fusion mechanism with a general back propagation structure with 3 layers. There are $4 \times 30$ nodes in the input layer for the confidence information from Table1 (30 class confidences of 4 face regions), followed by a hidden layer of 10 neurons. The output layer has 30 nodes for 30 face classes. The structure of the artificial neural network is shown in Figure 7.

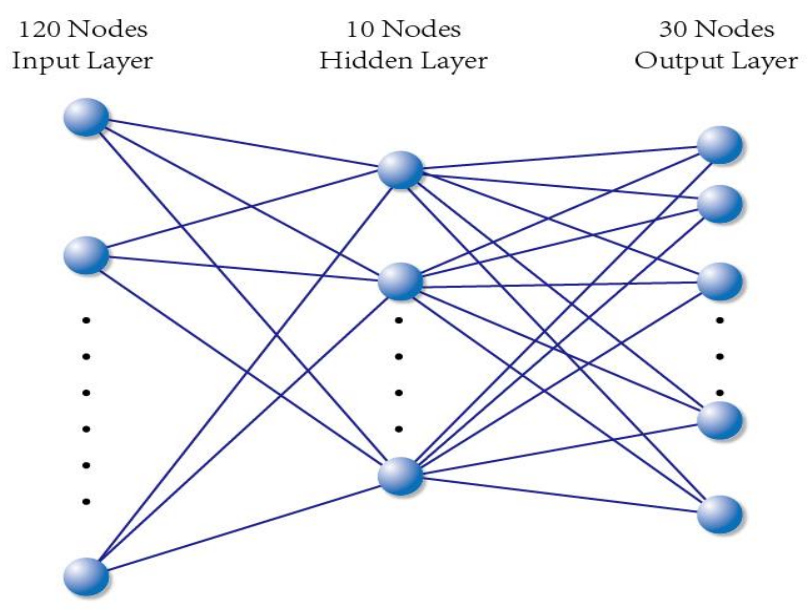

Figure 7. ANN Structure

We utilize TRAINLM (Levenberg-Marquardt) for back propagation network training function, LEARNGDM (Grads Descend on Momentum) for back propagation weight/bias learning function, MSE for performance function, and TANSIG as transfer function for the hidden layer and output layer. Some typical training process is illustrated in Figure 8.

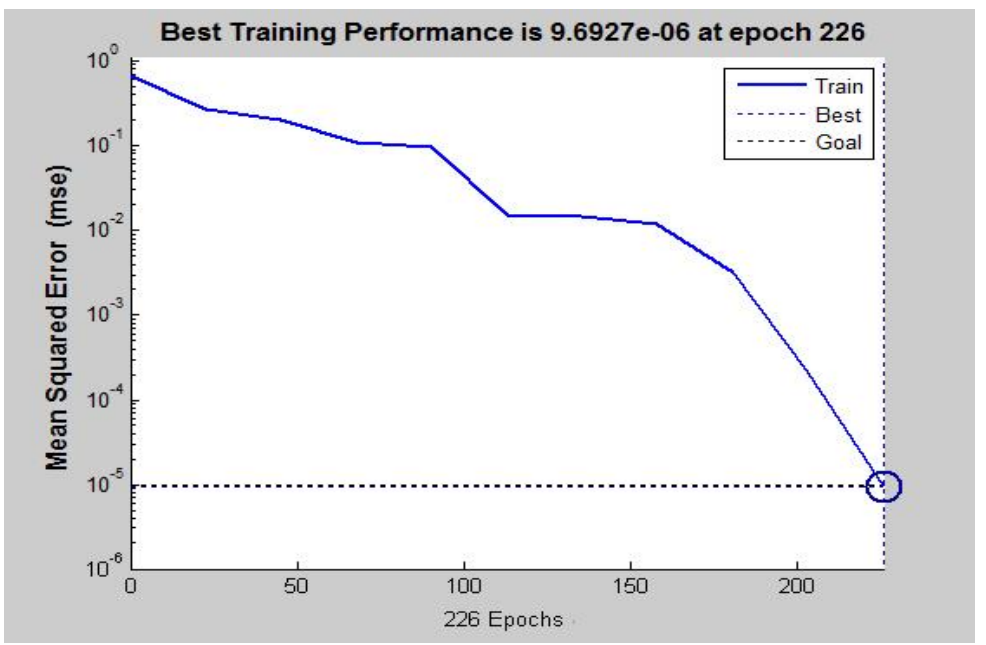

Figure 8. Some Typical Training Process (Training Goal Achieved) 
In test, the confidence table of each input image is computed as described in section 4.1 and the confidence data is rearranged as a feature vector $\vec{v}$ of length $4 \times 30$. Let $\vec{v}$ be the input of the artificial neuron network trained above, the index of output node with the max output gives the classification result.

\section{A Distributed Framework for Face Recognition}

In light of the data fusion mechanism introduced above, the whole face recognition system can be implemented with a distributed framework as illustrated in Figure 9, in which MBLBP features of each single face region are utilized to perform face classification in parallel. The final decision is given by an ANN based data fusion of the confidence information got from the SVM classification of each face region.

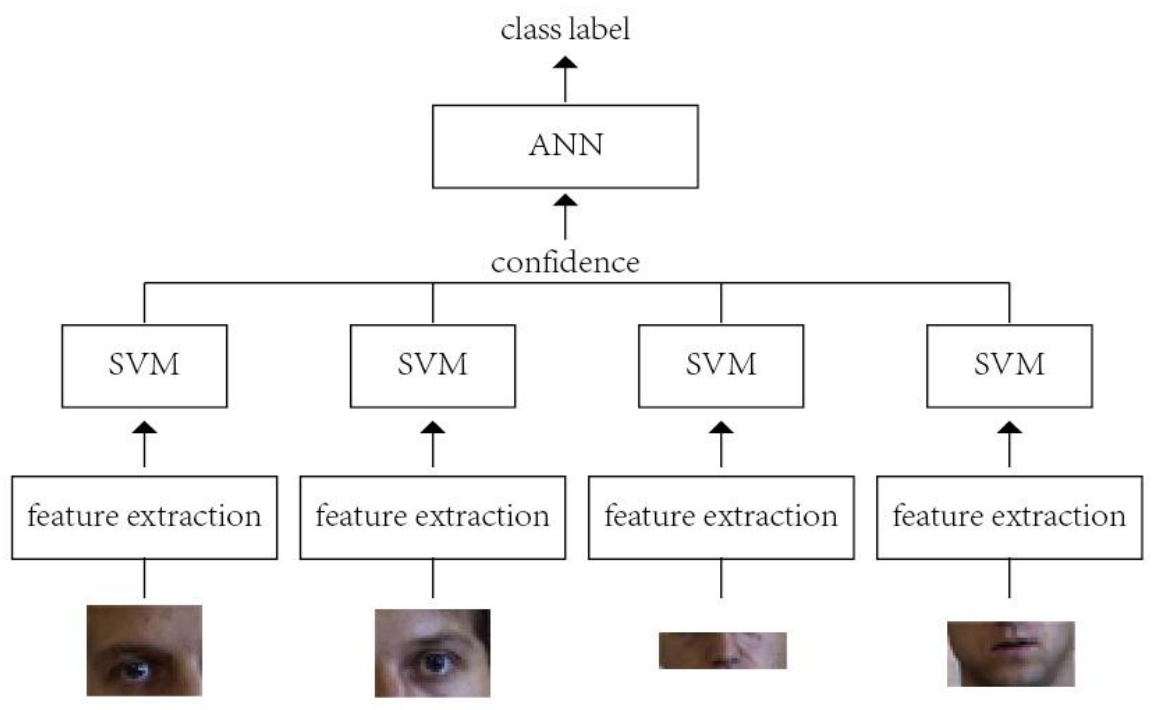

Figure 9. The Distributed Framework

In implementation, we can assign one separate processor for the subtask of computing the confidence information by using each of the 4 face regions, and then combine these confidence information to form the input vector for the neural network to do data fusion.

\section{Experimental Results}

In order to evaluate the distributed face recognition framework we present in this paper more objectively, two kinds of experiments are conducted here. In experiment-I, the accuracy of our system is compared with several other face recognition paradigms, such as LBP based method [3], and MB-LBP [5] based method with no fusion. The recognition rates are computed based on the average performance of a 5-fold cross validation, which are given in the second row of Table 2. To test the robustness of our system, we also use images under different illuminations with lights turned off (Figure 10) as new test set, and results are given in the third row of Table 2. 
Table 2. Recognition Rates

\begin{tabular}{|c|c|c|c|}
\hline & $\begin{array}{c}\text { Distributed data } \\
\text { fusion }\end{array}$ & LBP based method & $\begin{array}{c}\text { MBLBP+SVM with no } \\
\text { fusion }\end{array}$ \\
\hline $\begin{array}{c}\text { Cross } \\
\text { Validation }\end{array}$ & 84 & 86.4 & 90.8 \\
\hline New test set & 79.2 & 76.9 & 80.5 \\
\hline
\end{tabular}
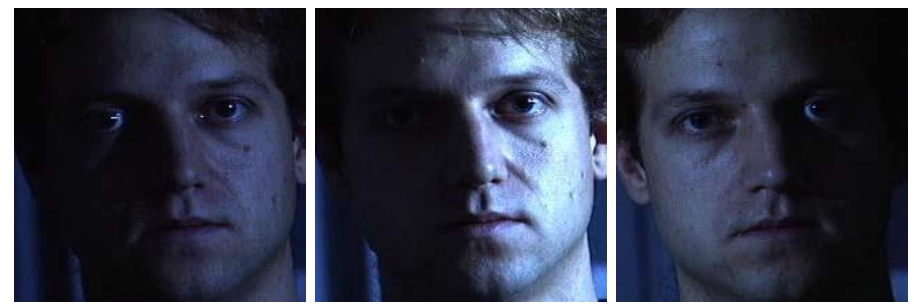

Figure 10. Test Images with Lights Off

As we can see from Table 2, in cross validation, all images in dataset are with lights turned on, our method achieves comparative results with LBP based method, while MB-LBP based method with no fusion performs best. When evaluating the new test set, our proposed method outperforms the LBP based method and achieves comparative results with MB-LBP based method with no fusion. As face regions become smaller, Illumination conditions in each face region is more likely to be uniform, this may indicate why our approach achieves better performance on the new test set.

Experiment-II is designed to verify the high efficiency of our distributed system. In experiment, we utilize Matlab distributed computing engine and assign a worker to each computation node (a computer in LAN). The runtime of our system are given in Figure 11 with the number of nodes from 1 up to 4 since we have totally 4 computation subtasks.

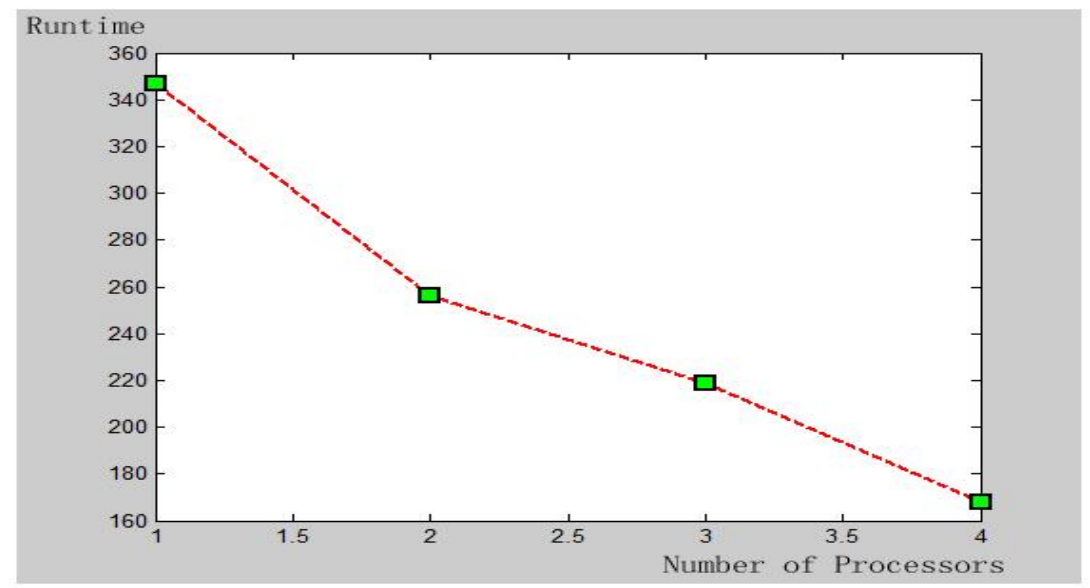

Figure 11. Runtime with the Number of Nodes from 1 up to 4

The speedup of our system versus the situation of non-distributed data fusion and MB$\mathrm{LBP}+\mathrm{SVM}$ with no fusion is given in Figure 12 with the number of nodes from 1 up to 4. 


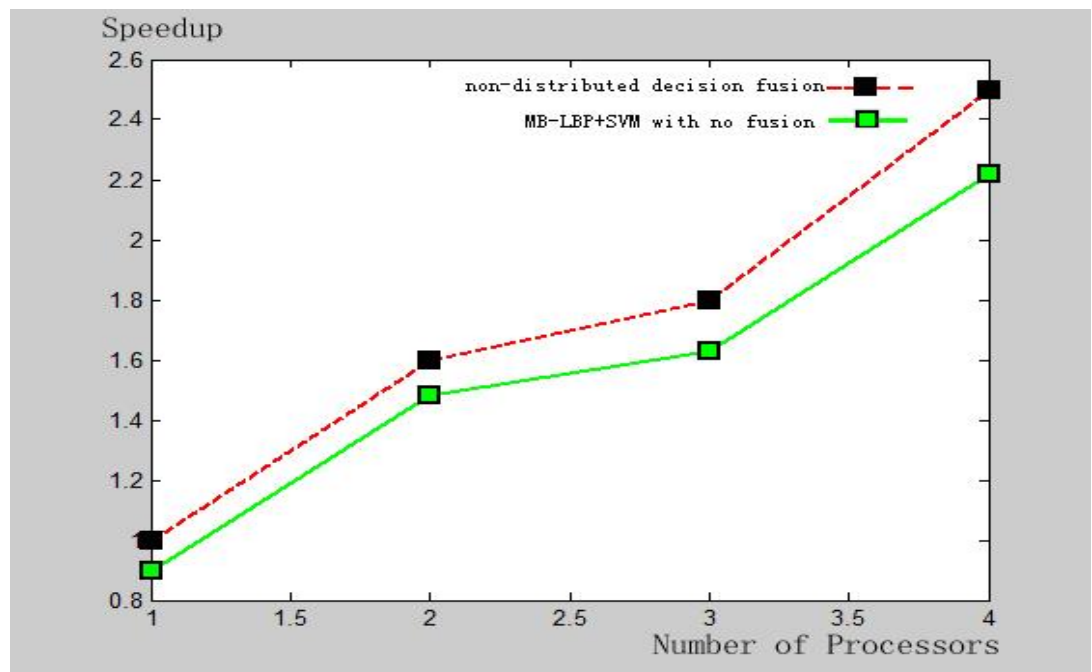

\section{Figure 12. Speedup Versus Non-distributed Data Fusion System and MB- LGBP+SVM with No Fusion}

\section{Conclusions and Future Works}

This paper presents a distributed face recognition framework based on multi-block local binary patterns and data fusion. Experiments are conducted on images from CMU PIE face database to verify the validation of our method by comparing the recognition efficiency and accuracy of our system with several other popular paradigms, and the results show that our approach can promote the efficiency of face recognition prominently while not losing accuracy in recognition performance.

However, the face regions in which the MB-LBP composite features are extracted are fixed and partitioned only by experience, the problem of how to partition them adaptively to make the illumination in one region more uniform still needs further consideration. And also the future work is to test the impact of different granularity of subtasks and the acceleration ratio with different feature vector length.

\section{Acknowledgements}

The authors would like to thank the Robotics Institute of Carnegie Mellon University for providing the CMU PIE database. The authors would also like to thank to Mr. Cheung for providing us with the distributed experimental environment.

\section{References}

[1] A. S. Tolba, A.H. El-Baz, and A.A. El-Harby, Face Recognition: A Literature Review, International Journal of Signal Processing, vol. 2, no. 88, (2006).

[2] R. Jafri and H. R. Arabnia, "A Survey of Face Recognition Techniques", Information Processing Systems, vol. 5, no. 41, (2009).

[3] T. Ahonen, A. Hadid and M. Pietikainen, "Face Recognition with Local Binary Patterns", Proceedings of the European Conference on Computer Vision, Prague, Czech, (2004), pp. 469-481.

[4] T. Ahonen, A. Hadid and M. Pietikainen, "Face Description with Local Binary Patterns: Application to Face Recognition", IEEE Trans. Pattern Anal. Mach. Intell., vol. 28, no. 2037, (2006).

[5] S. C. Liao, X. X. Zhu, Z. Lei and L. Zhang, "Learning Multi-Scale Block Local Binary Patterns for Face Recognition", Proceedings of IAPR/IEEE International Conference on Biometrics, Seoul, Korea, (2007) August, pp. 828-837.

[6] M. A. Turk and A. P. Pentland, "Eigenfaces for Recognition”, J. Cogn. Neurosci. vol. 3, no. 71, (1991). 
[7] H. Moon and P. J. Phillips, "Computational and Performance Aspects of PCA-based Face-Recognition Algorithms”, Perception, vol. 30, no. 303, (2001).

[8] L. Du, Z. Jia and L. Xue, "Human Face Recognition Based on Principal Component Analysis and Particle Swarm Optimization BP Neural Network", Proceedings of 3rd Conference on Natural Computation, vol. 3, (2007) August, pp. 287-291.

[9] Y. Q. Xu, B. C. Li and B. Wang, "Face Recognition by Fast Independent Component Analysis and Genetic Algorithm", Proc. of the 4th International Conference on Computer and Information Technology, (2004) September, pp. 194-198.

[10] P. N. Belhumeur, J. P. Hespanha and D. J. Kriegman, "Eigenfaces vs. Fisherfaces: Recognition Using Class Specific Linear Projection", IEEE Trans. Pattern Anal. Mach. Intell. vol. 19, no. 711, (1997).

[11] P. Penev and J. Atick, "Local Feature Analysis: A General Statistical Theory for Object Representation", Neural Systems, vol. 7, no. 477, (1996).

[12] C. Liu and H. Wechsler, "Gabor Feature Based Classification Using the Enhanced Fisher Linear Discriminant Model for Face Recognition”, IEEE Trans. Image Processing, vol. 11, no. 467, (2002).

[13] H. B. Kekre, S. D. Thepade and A. Maloo, "Face Recognition Using Texture Feartures Extracted Form Walshlet Pyramid", Int. J. on Recent Trends in Engineering \& Technology, vol. 5, no. 186, (2011).

[14] X. Y. Tan and B. Triggs, "Fusing Gabor and LBP Feature Sets for Kernel-based Face Recognition", 3rd International Workshop Analysis and Modelling of Faces and Gestures, (2007).

[15] W. F. Liu and Z. F. Wang, "Facial Expression Recognition Based on Fusion of Multiple Gabor Features", 18th International Conference on Pattern Recognition, vol. 3, (2006), pp. 536-539.

[16] T. Sim, S. Baker and M. Bsat, "The CMU Pose, Illumination, and Expression Database", IEEE Trans. Pattern Anal. Mach. Intell. vol. 25, no. 1615, (2003).

[17] C. W. Hsu, C. C. Chang and C. J. Lin, "A practical guide to support vector classification”, National Taiwan University, (2004).

[18] C. C. Chang and C. J. Lin, "LIBSVM: A Library for Support Vector Machines", available at http://www.csie.ntu.edu.tw/ cjlin/libsvm, (2001).

[19] P. J. Phillips, "Support Vector Machines Applied to Face Recognition", Proceedings of the 11th International Conference \& Workshop on Emerging Trends in Technology, (2011), pp. 803-809.

[20] A. K. Jain, J. C. Mao and K. M. Mohiuddin, “Artificial Neural Networks: A Tutorial”, Computer, vol. 29, no. 31, (1996).

\section{Authors}

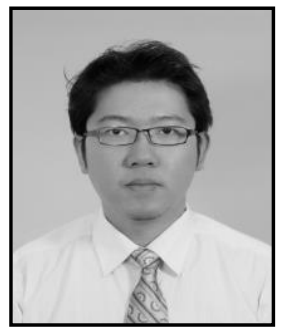

Zheng Zhang, received his BEng degree in Computer Science and Technology from Jilin University in 2002, MEng degree in Computer Application from Nankai University in 2007, and PhD degree in Computer Application from Tianjin University in 2010. He is currently a Lecturer with the Department of Software Engineering of the School of Computer Science and Software Engineering at Tianjin Polytechnic University in China. He has published over 10 international journal and conference papers, books. His research interests are in the areas of computer vision, pattern recognition, and 3D computer graphics.

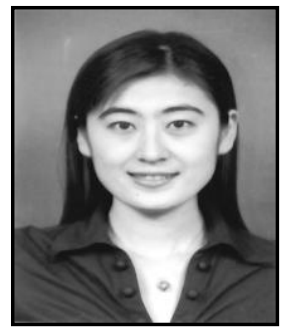

Yan Guo, received her BEng degree in Tianjin Conservatory of Music University in 2002, MEng degree in Tianjin Normal University in 2008. She is currently a Lecturer with School of Computer Software at Tianjin University in China. 


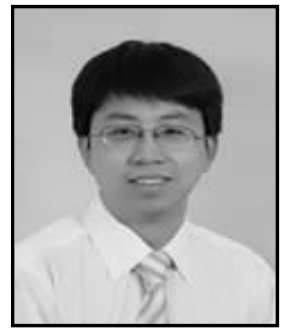

Guozhi Song, received his BEng degree in Computer Science and Technology from Harbin Institute of Technology, and both his MSc and $\mathrm{PhD}$ degrees from the School of Electronic Engineering and Computer Science at Queen Mary, University of London. He is currently a Lecturer with the Department of Networks of the School of Computer Science and Software Engineering at Tianjin Polytechnic University in China. 
International Journal of Database Theory and Application Vol.7, No.4 (2014) 